\title{
CASE REPORT OF A PATIENT WITH A CLINICAL PICTURE SUGGESTIVE OF CANDLE SYNDROME
}

Nicole Carvalho Xavier Micheloni da Silva ${ }^{1}$, Ana Flávia da Silva Pina ${ }^{1}$, Amanda Aterje Pelloso ${ }^{1}$, Ana Paula Sakamoto ${ }^{1}$, Melissa Mariti Fraga ${ }^{1}$, Daniela Gerent Petry Piotto ${ }^{1}$, Maria Teresa Terreri ${ }^{1, *}$

1. Universidade Federal de São Paulo, São Paulo (SP), Brazil.

*Corresponding author: teterreri@terra.com.br

\section{BACKGROUND}

Chronic atypical neutrophilic dermatosis with lipodystrophy and elevated temperature (CANDLE) syndrome is an autoinflammatory disease (AID) characterized by recurrent fever, skin lesions, lipodystrophy and multisystem inflammation. Mutations lead to dysfunction of proteasome-immunoproteasome system and state of continuous inflammation.

\section{CASE REPORT}

Seven days old patient, male, presented with skin lesions (initially papules, then pustules, some with central necrosis), which spontaneously improved in 2 months (Figs. 1 and 2). Skin biopsy revealed neutrophilic dermatosis with dermal mucinosis. Completely improved with prednisolone $1 \mathrm{mg} / \mathrm{kg} /$ day and relapsed with corticosteroid weaning. At 6 months old, he developed daily fever; at 8 months old, he started hyperemia and periorbital edema, with low weight-height gain (without lipodystrophy), delayed neuropsychomotor development, besides microcytic hypochromic anemia, increased ESR and CRP, hyperferritinemia, elevated liver and muscle enzymes and normal myelogram. Other differential diagnoses were excluded. Considering the hypothesis of CANDLE syndrome, colchicine at $0.02 \mathrm{mg} / \mathrm{kg} / \mathrm{day}$ and prednisolone at $0.5 \mathrm{mg} / \mathrm{kg} /$ day doses were initiated, while we are waiting for the AID panel followed by improvement of fever and skin lesions.

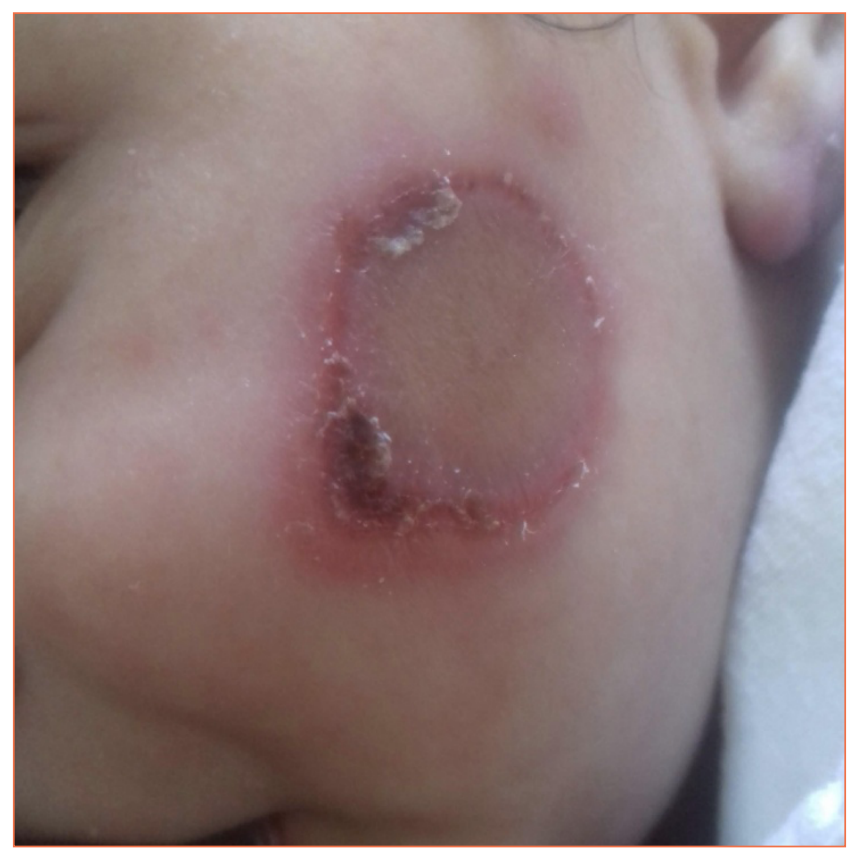

Figure 1. Skin lesion on face. 


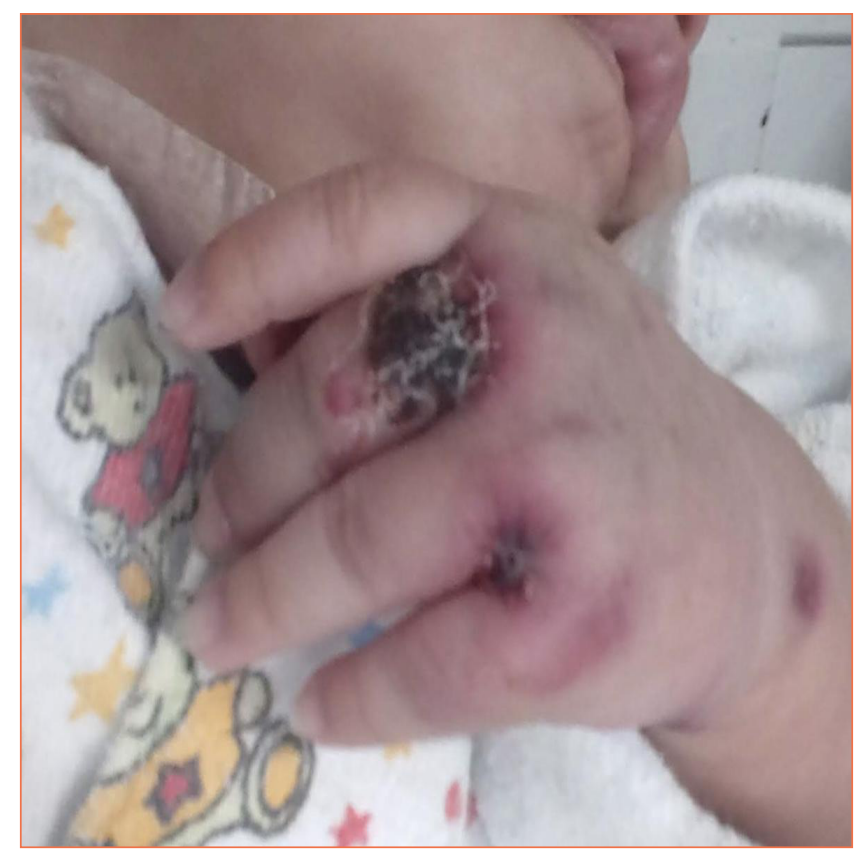

Figure 2. Skin lesion with central necrosis.

\section{CONCLUSION}

CANDLE is an AID suspected in an infant, boy, with fever and skin lesions compatible with neutrophilic dermatosis. Genetic confirmation is still pending. 